\title{
Gas chromatographic/mass spectrometric and microbiological analyses on irradiated chicken
}

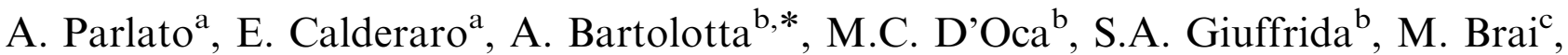

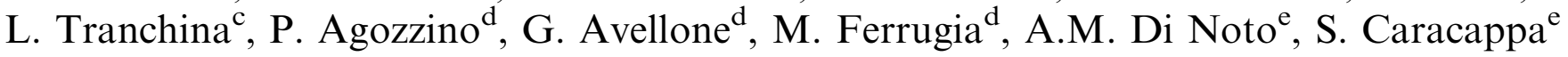 \\ ${ }^{a}$ Dipartimento di Ingegneria Nucleare, Università di Palermo, Viale delle Scienze, Edificio 6, 90100 Palermo, Italy \\ ${ }^{\mathrm{b}}$ Dipartimento Farmacochimico, Tossicologico e Biologico, Universitá di Palermo, via Archirafi 32, 90123 Palermo, Italy \\ ${ }^{\mathrm{c}}$ Dipartimento di Fisica e Tecnologie Relative, Università di Palermo, Viale delle Scienze, Edificio 18, 90100 Palermo, Italy \\ ${ }^{\mathrm{d}}$ Dipartimento di Chimica e Tecnologie Farmaceutiche, Università di Palermo, via Archirafi 32, 90123 Palermo, Italy \\ 'Istituto Zooprofilattico Sperimentale della Sicilia "A.Mirri”, Palermo, Italy
}

\begin{abstract}
Ionizing radiation is widely used as treatment technique for food preservation. It involves among others reduction of microbial contamination, disinfestations, sprout inhibition and extension of shelf life of food. However, the commercialization of irradiated food requires the availability of reliable methods to identify irradiated foodstuffs. In this paper, we present results on the application to irradiated chicken of this method, based on the detection, in muscle and skin samples, of the peaks of ions $98 \mathrm{Da}$ and $112 \mathrm{Da}$, in a ratio approximately 4:1, typical of radiation induced 2-dodecylcyclobutanones (2-DCB). Aim of the work was also to study the time stability of the measured parameters in samples irradiated at 3 and $5 \mathrm{kGy}$, and to verify the efficacy of the treatment from a microbiological point of view. Our results show that, one month after irradiation at $3 \mathrm{kGy}$, the method is suitable using the skin but not the muscle, while the measured parameters are detectable in both samples irradiated at $5 \mathrm{kGy}$. The microbial population was substantially reduced even at $3 \mathrm{kGy}$.
\end{abstract}

(C) 2007 Elsevier Ltd. All rights reserved.

Keywords: Irradiated food; Gas chromatography; Radiation treatment

\section{Introduction}

In recent years the interest in food safety is grown more and more, not only from the administration, the researchers and the food industry side, but mainly from the consumers point of view.

The microbiological contamination represents a serious problem, and the strategies aimed to prevent and to reduce its risk are in continuous evolution. The irradiation of foods against the microbiological risk surely is an innovative approach in comparison to the traditional methods as chemical fumigation.

Radiation treatment is carried out with gamma or electron beams, with energy and dose values that do not modify nutritional properties of irradiated food, do not

\footnotetext{
*Corresponding author. Tel.: + 390916162323 ; fax: + 390916169999.

E-mail address: antonio.bartolotta@unipa.it (A. Bartolotta).
}

produce toxical effects and do not induce radioactivity in food itself (WHO, 1987).

Irradiation with doses lower than $10 \mathrm{kGy}$ (WHO, 1999) is widely used as treatment technique for food preservation. It allows to drastically reduce the microbial contamination, to disinfect from insects, to prevent sprouting; a reduction of food spoilage and an extension of the shelf life of food can be also achieved. However, the commercialization of irradiated food requires the availability of reliable methods to identify irradiated foodstuffs. The European Community has validated standard biological, physical and chemical methods, each one specific for some food typology; among them, the gas chromatography/mass spectrometry is recommended for the detection of irradiated food containing fat.

The main goal of the research discussed in this paper was to investigate the application of the identification method (EN 1785, 1996) based on gas chromatography and mass 
spectrometry (GC/MS) in irradiated chicken. In particular we have studied if the irradiation treatment was detectable within the typical shelf life of the chicken, and in which part of it (skin or muscle), as a function of dose and storage conditions. Moreover, the effect of ionizing radiation on the microbiological population was investigated.

\section{Materials and methods}

Among the 2-alkylcyclobutanones produced in irradiated meat, we have chosen to analyze the 2-dodecylcyclobutanone (2-DCB), which is formed from palmitic acid during irradiation.

\subsection{Fat extraction and purification of extracts obtained}

Chickens were purchased at local stores, packed in aluminium boxes, and stored at $(0-4){ }^{\circ} \mathrm{C}$. For each analysis (carried out on two different chickens), the lipids were extracted from a sample of $20 \mathrm{~g}$ composed by muscle $(15 \mathrm{~g})$ and skin $(5 \mathrm{~g})$ of chicken, that is approximately the natural composition of chicken meat, with an average fat content between $1 \%$ and $4 \%$; for comparison purposes, sample of muscle alone or skin alone were investigated. Each sample was homogenized and mixed with $20 \mathrm{~g}$ of anhydrous sodium sulfate. Each lipid fraction, $200 \mathrm{mg}$ each, was purified by solid phase extraction on a column Florisil $\mathbb{R}$ deactivated. The hexane extracts, expecting to contain 2-DCB, was concentrated to a volume of $5 \mathrm{ml}$ at the temperature of $40^{\circ} \mathrm{C}$ using a rotary vacuum evaporator, and further concentrated to complete dryness by nitrogen, and resuspended with $n$-hexane. A standard 2-DCB solution was prepared by solving 2-DCB in $n$-hexane to a final concentration of $0.5 \mu \mathrm{g} / \mathrm{ml}$.

\subsection{Separation and identification of 2-DCB}

The GC/MS analysis was carried out on Varian Saturn 3-Ion Trap System spectrometer. The used capillary column was a J\&W DB 5MS. The following conditions were applied: helium was used as the carrier gas at flow rate of about $1 \mathrm{ml} / \mathrm{min}$, the transfer line and ion trap temperature was $180^{\circ} \mathrm{C}$; the injector temperature was fixed at $250^{\circ} \mathrm{C}$; the column temperature program was: $1 \mathrm{~min}$ at $55^{\circ} \mathrm{C}$ followed by a ramp rate of $15^{\circ} \mathrm{C} / \mathrm{min}$ up to $300^{\circ} \mathrm{C}$ held for $10 \mathrm{~min}$. The ion trap mass spectrometer parameters were: electron ionization mode, ionizing energy $70 \mathrm{eV}$, mass range $30-200 \mathrm{Da}$ and the scan frequency was $4 \mathrm{scans} / \mathrm{s}$; injected volume $1 \mu \mathrm{L}$. The collected data were processed with dedicated software of the instrument. For the identification of 2-DCB we have followed the protocol EN 1785 [3]. In order to improve sensitivity, the selected ions monitoring (SIM) mode was also used, recording ions with 98 and $112 \mathrm{~m} / \mathrm{z}$, with a Hewlett-Packard GC/MS system.

\subsection{Irradiation}

Aliquots of $100 \mathrm{~g}$ of chicken, constituted by muscle and skin, have been irradiated at 3 and $5 \mathrm{kGy}$, with a ${ }^{60} \mathrm{Co}$ panoramic irradiator IGS-3. The dose values were calculated using irradiation time and the dose rate $(2.00 \mathrm{kGy} / \mathrm{h})$ measured with the Fricke dosimetry.

\subsection{Microbiological analyses}

The following microbiological analyses were conducted on one sample of chicken before and one after irradiation (storage temperature $0-4{ }^{\circ} \mathrm{C}$ ), according to international protocols: total aerobic microbial enumeration at $30^{\circ} \mathrm{C}$, enumeration of coliforms and $E$. coli, enumeration of coagulase-positive Staphylococci, Enterococcus spp. on bile aesculin agar, sulphite-reducing Clostridia, detection of Salmonella spp.

Table 1

The table shows the results of the bacteriological examination on the chicken samples before $(1 \times 30 \mathrm{~g})$ and after $(1 \times 30 \mathrm{~g})$ the radiation treatment at $3 \mathrm{kGy}$

\begin{tabular}{lll}
\hline Bacteriological test & Unirradiated $(\mathrm{cfu} / \mathrm{g})$ & Irradiated $(\mathrm{cfu} / \mathrm{g})$ \\
\hline CMT & $2.7 \times 10^{8}$ & $1.1 \times 10^{5}$ \\
Coliforms & $1.2 \times 10^{2}$ & $<1 \times 10^{1}$ \\
E. coli & $8.0 \times 10^{1}$ & $<1 \times 10^{1}$ \\
Staphylococci coagulase-positive & $<10 \times 10^{1}$ & $<10 \times 10^{1}$ \\
Enterococcus spp. & $1.4 \times 10^{3}$ & $6.0 \times 10^{1}$ \\
Clostridia s.r. & $<1 \times 10^{1}$ & $<1 \times 10^{1}$ \\
Salmonella spp. & Not detectable & Not detectable \\
\hline
\end{tabular}
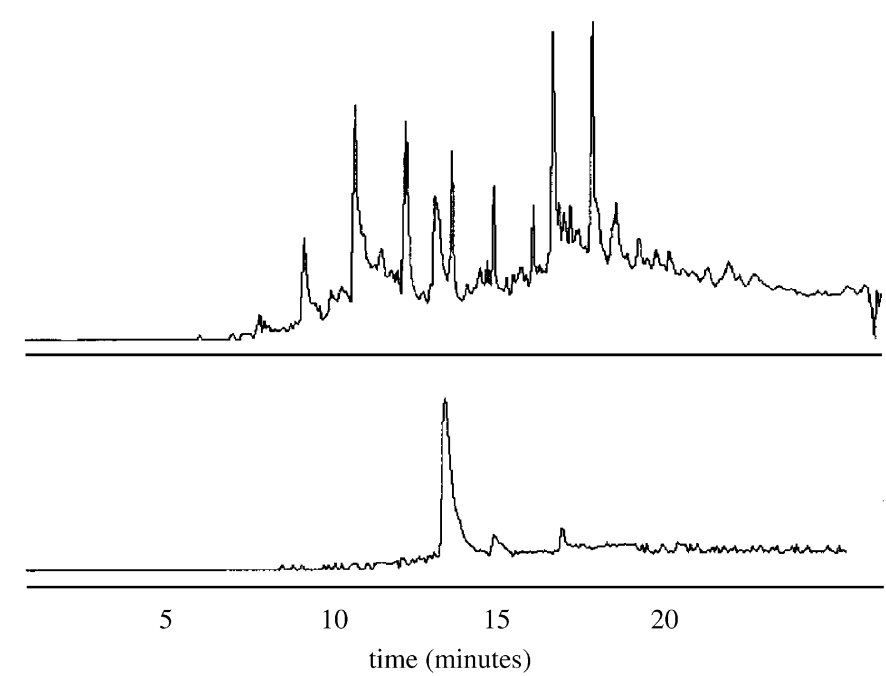

Fig. 1. Chromatograms obtained for the detection of 2-dodecylcyclobutanone (selected ions monitoring of the ions 98 and $112 \mathrm{Da}$ ) in a sample of chicken irradiated at $5 \mathrm{kGy}$ (up), and in a solution containing the standard 2-DCB (down). 


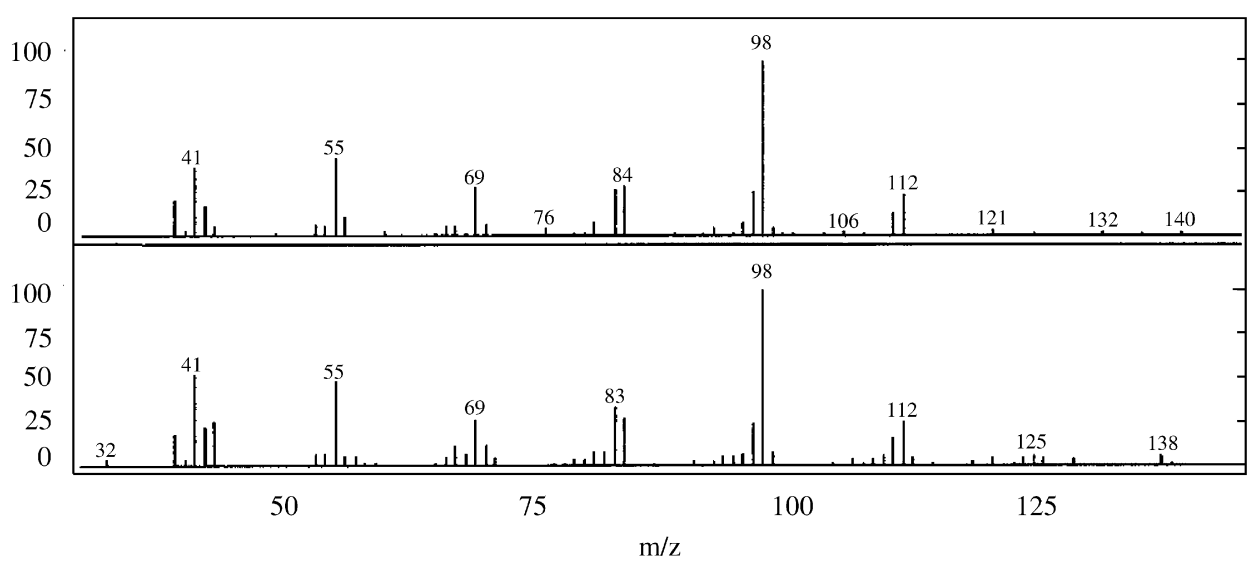

Fig. 2. Mass spectrum of the peak corresponding at the retention time of 2-DCB extracted from a sample of chicken irradiated at $5 \mathrm{kGy}$ (up), and the corresponding spectrum of the 2-DCB standard (down).

\section{Results and discussion}

The results of the bacteriological examination on the chicken sample after the radiation treatment at $3 \mathrm{kGy}$ (Table 1) emphasize strong reduction of the contaminating microbiological flora; the residual microbial flora concentration is attributable to the dose used in this preliminary experiment, relatively low. It is important to mention, however, that the radiation treatment is not allowed to be used to improve originally spoiled food.

As for the GC/MS analysis, it was preliminary carried out on the 2-DCB standard to evaluate its retention time, that was found to be at $13.5 \mathrm{~min}$; the corresponding mass spectrum showed peaks of ions 98 and $112 \mathrm{Da}$ in the ratio 4:1, approximately. Moreover, the extract ions chromatogram of unirradiated samples of muscle and skin of chicken did not show any specific signal at the retention time attended for 2-DCB.

On the other hand, the 2-DCB, expected from chicken lipids after irradiation, was clearly detected, at expected retention time $13.5 \mathrm{~min}$ (Fig. 1); in the corresponding mass spectrum both ions 98 and $112 \mathrm{Da}$ in the ratio 4:1 were present (Fig. 2).

Comparable results were obtained with the sample of muscle alone (without skin) irradiated at 3 or $5 \mathrm{kGy}$. These results show that the extraction and analysis procedure set up by us allow to correctly discriminate samples of irradiated chicken from unirradiated ones, at least soon after irradiation treatment.

To verify its applicability as identification method even on chicken samples stored after irradiation, the GC/MS analysis was carried out also on the following samples (storage temperature between -20 and $0^{\circ} \mathrm{C}$ ):

Sample a: skin irradiated at $3 \mathrm{kGy}$ and stored for 30 days; Sample b: muscle irradiated at $3 \mathrm{kGy}$ and stored for 30 days;

Sample c: muscle irradiated at $5 \mathrm{kGy}$ and stored for 40 days.
In the sample a (skin without muscle), the presence of the 2-DCB could be clearly detected and confirmed by mass spectrum; in sample b (muscle without skin) irradiated and stored as sample a, the 2-DCB was instead almost undetectable. This result indicates the importance of the presence of the skin in the sample to be analyzed, because the skin contains a high concentration of palmitic acid, precursor of 2-DCB. Finally, the 2-DCB was detectable in the muscle alone (sample c) at the higher dose of $5 \mathrm{kGy}$ after 40 days storage.

\section{Conclusion}

The main conclusion of this preliminary work is that the GC/MS technique, recommended by the European Standard 1785 as identification method of irradiated food containing fat (EN 1785, 1996), can be successfully applied on chicken irradiated at $3 \mathrm{kGy}$, soon after irradiation (skin or muscle). After a storage time of 30 days, the identification is still achievable provided that the investigation is carried out on the skin, whereas on samples without skin the technique is applicable only at higher doses.

\section{Acknowledgments}

This work was partially supported by the Università di Palermo and by the Istituto Zooprofilattico Sperimentale della Sicilia.

\section{References}

EN 1785, 1996. Foodstuffs-detection of irradiated food containing fat-gas chromatographic/mass spectrometric analysis of 2-alkylcyclobutanones. European Committee for Standardization, Brussels, Belgium.

WHO (World Health Organization), 1987. International Consultive group on food irradiation (ICGFI). Task Force Meeting on the Use of Irradiation to Ensure Hygienic Quality of Food, 14-18 July, Vienna, Austria, WHO, Geneva, 1987 (WHO/EHE/FOS/87.2).

WHO, 1999. High-dose irradiation: wholesomeness of food irradiated with doses above $10 \mathrm{kGy}$. WHO Technical Report Series No. 890, WHO, Geneva, 1999. 